The Agriculturists 15(1):156-169(2017) ISSN 2304-7321 (Online), ISSN 1729-5211 (Print)

\title{
Effect of Arbuscular Mycorrhizal Fungi on the Tolerance to Sodium Chloride Levels, and on Growth and Yield of Lentil (Lens culinaris)
}

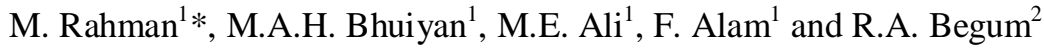 \\ ${ }^{1}$ Soil Microbiology Laboratory and ${ }^{2}$ Soil Science Division, Bangladesh Agricultural Research \\ Institute, Gazipur-1701, Bangladesh \\ *Corresponding author and Email: mominurssd13@bari.gov.bd, mominurbau@gmail.com
}

Received: 09 December 2016

Accepted: 12 June 2017

\begin{abstract}
A pot experiment was carried out in the nethouse of Soil Science Division, Bangladesh Agricultural Research Institute, Joydebpur, Gazipur during 2014-2015 through 2015-2016. The design of the experiment was factorial randomized completely block design with 4 replications. The objectives of the study were to evaluate the potential of Arbuscular mycorrhization (AM) on the germination, yield and yield attributes of lentil treated with different concentration of sodium chloride $(\mathrm{NaCl})$. Five $\mathrm{NaCl}$ treatments $(0,1,2,3$ and $4 \%)$ possessed $\mathrm{NaCl}$ level as the first factor that were treated with soils before sowing of lentil seeds overriding or pivotal pulse crop in Bangladesh. The second factor consists of mycorrhizal and non-mycorrhizal treatments. Mycorrhizal plants showed better performance in terms of germination \%, yield and yield contributing characters than non-mycorrhizal plants. With increasing $\mathrm{NaCl}$ concentration germination \%, yield and yield contributing characters in the rhizosphere soil, decreased significantly $(\mathrm{p}<0.01)$. Interaction effects of mycorrhizal inoculation and $\mathrm{NaCl}$ on germination \%, growth and yield of lentil were appeared to be statistically nonsignificant. The highest germination (96.25\% in $2014-2015$ and $92.50 \%$ in $2015-2016$ ), seed yield (6.45 $\mathrm{g} \mathrm{pot}^{-1}$ in 2014-2015 and 5.89 $\mathrm{g} \mathrm{pot}^{-1}$ in 2015-2016), and stover yield (9.55 $\mathrm{g} \mathrm{pot}^{-1}$ in 2014-2015 and $8.58 \mathrm{~g} \mathrm{pot}^{-1}$ in 2015-2016) was found in $\mathrm{NaCl} 0 \%+\mathrm{AM}$ treatment. The lowest germination \%, seed yield and stover yield was found in $\mathrm{NaCl} 4 \%$ treatment. Mycorrhizal inoculation increased seed yield on an average by $31.85 \%$ during $2014-2015$ and $63.71 \%$ during $2015-2016$, and increased stover yield on an average by $48.56 \%$ during 2014-2015 and 63.55\% during 2015-2016 over non-mycorrhizal inoculation. Therefore, it can be concluded that mycorrhizal inoculation increases germination \%, growth and yield of lentil over non-mycorrhizal inoculation.
\end{abstract}

Keywords: Mycorrhizal inoculation, germination, lentil, sodium chloride, yield.

\section{Introduction}

Soil salinity is one of the major environmental hazards of the present world including Bangladesh greatly affecting agricultural production as well as food security. Abiotic and biotic stresses hamper the production of our cultivated land at an alarming rate. Among the abiotic stresses salt stress has a greater impact on farmlands worldwide. It is reported that about $7 \%$ of the total land on earth and $20 \%$ of the total arable land are affected by high salt content (Mali et al., 2012). In view of another projection, $2.1 \%$ of the global dry land agriculture is affected by salinity. Over $30 \%$ of the cultivable area of Bangladesh lies in the coastal and 
offshore zones. Out of 2.86 million hectares of coastal and offshore lands, about 1.06 million hectares are affected by varying degrees of salinity (SRDI, 2010). The area under salinity is increasing with time (from $0.83 \mathrm{~m}$ ha to $1.06 \mathrm{~m}$ ha in 36 years; SRDI, 2010) due to rise in sea water level with increased global temperature. The other main three causes are tidal flooding, salinity intrusion and capillary rise of saline water.

In the world associated agricultural production losses were estimated to be close to $\$ 12$ billion per year due to soil salinization. Furthermore, the salinized areas are increasing at a rate of $10 \%$ annually for various reasons. It has been estimated that more than $50 \%$ of the arable land would be salinized by the year 2050 (Jamil et al., 2011). On the other hand the human race is increasing and is believed to reach 8.3 billion by 2030. It is difficult to feed this increasing population as the productive land is decreasing day by day. Agriculture is the most important sector of Bangladesh economy. Usually $30-50 \%$ yield losses occur depending on the level of soil salinity.

Researchers are trying to their level best to find out suitable techniques to combat these concerning problems ie. looking for an alternative to bring the uncultivated land under cultivation. But these techniques seem to be very costly and unaffordable to underdeveloped countries, whereas microorganisms specially arbuscular mycorrhiza (AM) have the potential to reduce the sodium and chloride toxicity in crops which could be a cost effective environmental friendly option that is available in a shorter time frame. Arbuscular mycorrhizas (AM) are pervasive and they are found in $80 \%$ of vascular plant families in existence today and fungi belonging to the order glomeromycota. AM have been shown to promote plant growth (Hameed et al., 2014), enhance nutrient uptake such as nitrogen, phosphorus, magnesium, and micronutrients from the soil (Evelin et al., 2012), improve soil structure, and also able to enhance plant tolerance under different stresses such as drought and salinity (Wu et al., 2014). Plants treated with AM fungi have shown to have enhanced the growth and yield, and maintains the osmotic and ionic balance to a normal level so that plants will thrive well under these stress conditions (Hameed et al., 2014).

Lentil (Lens culinaris) var. BARI Masur-5 belongs to the family Fabaceae and is commonly used as an important pulse crop in Bangladesh. In 2013-2014, about 136,695 ha of land are under lentil cultivation and the total production is about 157,422 metric tons and in 2014-2015, about 145,493 ha of land is under lentil cultivation and the total production is about 167,261 metric tons (BBS, 2015). An ideal sustainable agricultural system is one which maintains and improves human health, benefits producers and consumers both economically and spiritually, protects the environment, conserve ecosystem, sustain soil health and produces enough food for an increasing world population. Plant associated microorganisms ie. arbuscular mycorrhiza can play an important role in conferring resistance to alleviating salinity stresses in plants. Taking the current leads available, concerted future research is needed to have an appraisal or summing-up of the present state of land areas affected by salinity. Therefore, the overall aim of the investigation was to evaluate the potential of arbuscular mycorrhizal inoculation on seed germination $(\%)$, growth and yield of lentil treated with different concentration of $\mathrm{NaCl}$.

\section{Materials and Methods}

\subsection{Seed collection and Soil preparation}

The during rabi season from December, 2014 through March, 2015 and December, 2015 through experiment was carried out March, 2016 in the net house of Soil Science Division, BARI, Joydebpur, Gazipur $\left(23^{\circ} 59^{\prime} 38^{\prime \prime} \mathrm{N}\right.$ latitude, $90^{\circ}$ 24'89" E longitude and $8.4 \mathrm{~m}$ elevation). Seeds of lentil (BARI Masur-5) were collected from Pulse Research Centre, BARI, Gazipur. The silted (sandy clay loam) soils were collected from the bank of Turag river at Kodda, Gazipur 
mixed with cowdung at 5:1 ratio and was used as the potting media. Each pot $(28 \mathrm{~cm}$ in diameter and $23 \mathrm{~cm}$ in height) was filled with approximately $8-\mathrm{kg}$ soil leaving upper 3 inches of pot vacant to facilitate watering. The $\mathrm{pH}$ of cowdung was 6.7 and the nutrient contents were: organic matter $14.1 \%, \mathrm{~N} 0.8 \%, \mathrm{P} 1.26 \%, \mathrm{~K}$ $0.88 \%$, Ca $1.55 \%, \mathrm{Mg} 0.82 \%, \mathrm{~S} 0.62 \%$, Fe $0.25 \%$ and $\mathrm{Mn} 0.112 \%$. The physical and chemical properties of the soil were presented in Table 1. The soil contained 12 AM $\left(100^{-1} \mathrm{~g}\right.$ soil $)$ spores of indigenous mixed AM fungal species and the experiment was conducted under unsterilized soil condition.

\subsection{Soil analysis}

Soil $\mathrm{pH}$ was measured by a combined glass calomel electrode. Organic carbon was determined by Wet Oxidation Method. Total N was determined by modified Kjeldahl method. Calcium, $\mathrm{K}$ and $\mathrm{Mg}$ were determined by $\mathrm{NH}_{4} \mathrm{OAc}$ extraction method. Copper, $\mathrm{Fe}, \mathrm{Mn}$ and $\mathrm{Zn}$ were determined by DTPA extraction followed by AAS reading. Boron was determined by $\mathrm{CaCl}_{2}$ extraction method. Phosphorus was determined by Modified Olsen method (Neutral + Calcareous soils). Sulphur was determined by $\mathrm{CaH}_{4}\left(\mathrm{PO}_{4}\right)_{2} \cdot \mathrm{H}_{2} \mathrm{O}$ extraction followed by turbidimetric method with $\mathrm{BaCl}_{2}$.

\subsection{Fertilizer application}

Chemical fertilizers @ $11.53 \mathrm{mg} \mathrm{N}: 9.9$ mg P: $11.55 \mathrm{mg} \mathrm{K}: 6.18 \mathrm{mg} \mathrm{S:} 0.60 \mathrm{mg}$ Zn: $0.37 \mathrm{mg} \mathrm{B}$ : $0.17 \mathrm{mg} \mathrm{Mo} \mathrm{kg}^{-1}$ soil was applied (BARC, 2012). Urea, TSP, MoP, Gypsum, $\mathrm{ZnSO}_{4}$, Boric acid and $\mathrm{Na}_{2} \mathrm{MoO}_{4}$ were used as a source of $\mathrm{N}, \mathrm{P}, \mathrm{K}$, $\mathrm{S}, \mathrm{Zn}, \mathrm{B}$ and Mo, respectively. Total amount of TSP, MoP, Gypsum, $\mathrm{ZnSO}_{4}$, Boric acid, $\mathrm{Na}_{2} \mathrm{MoO}_{4}$ and half of $\mathrm{N}$ was broadcast and incorporated during final pot preparation and remaining $\mathrm{N}$ was applied in two equal splits at 15 and 35 days after sowing.

\subsection{Preparation of $\mathrm{NaCl}$ solution and Mycorrhizal inoculum}

Different concentrations of $\mathrm{NaCl}$ solution was prepared according to experimental design and $250 \mathrm{~mL}$ of each $\mathrm{NaCl}$ solution was applied in each pot with irrigation water before sowing of lentil seed. The developed soil salinity was within the range of 1.04 to $3.75 \mathrm{dSm}^{-1}$.

The AM inoculum was prepared from the roots and rhizosphere soils of sorghum. Mycorrhizal species was originally isolated from different AEZs, using the wet sieving and decanting method (Gerdemann and Nicolson, 1963). The spores were left to multiply for 6 months on sorghum plants using unsterilized soil, collected from the same site, in the net house of Soil Science Division, BARI. Plants were irrigated with tap water as needed. A mixture of infected sorghum root and soil which contained spores was used as mycorrhizal inoculum. The soil based AM fungal inoculum containing $150 \mathrm{~g}$ of rhizosphere soil (approximate $209.67 \pm 5.5$ spores/100 g soil) and infected sorghum root fragments with a minimum infection level was inoculated to each mycorrhizal pot. Figure 1 represents different mycorrhizal spore identified in the Soil Microbiology Laboratory, Soil Science Division, BARI and used for the experiment. The mycorrhizal inoculum were first placed in each pot at $3-5 \mathrm{~cm}$ depth and was covered with a thin soil layer of $1 \mathrm{~cm}$ immediately prior to the seed sowing of lentil to facilitate fungal colonization of plant roots.

\subsection{Design of experiment and treatments}

The experiment was designed in factorial RCBD with 10 treatments combination and 4 replications. Twenty seeds were sown in each pot at $1 \mathrm{~cm}$ soil depth. The treatment was sustained with 11-14 vigorous seedlings in mycorrhizal and non-mycorrhizal pot and the other seedlings were removed from the pot. The 10 treatment combinations were: $\mathrm{T}_{1}: \mathrm{NaCl} 0 \%$, $\mathrm{T}_{2}: \mathrm{NaCl} 0 \%+\mathrm{AM}, \mathrm{T}_{3}: \mathrm{NaCl} 1 \%, \mathrm{~T}_{4}: \mathrm{NaCl} 1 \%$ $+\mathrm{AM}, \mathrm{T}_{5}: \mathrm{NaCl} 2 \%, \mathrm{~T}_{6}: \mathrm{NaCl} 2 \%+\mathrm{AM}, \mathrm{T}_{7}$ : $\mathrm{NaCl} 3 \%, \mathrm{~T}_{8}: \mathrm{NaCl} 3 \%+\mathrm{AM}, \mathrm{T}_{9}: \mathrm{NaCl} 4 \%$ and $\mathrm{T}_{10}: \mathrm{NaCl} 4 \%+\mathrm{AM}$.

\subsection{Determination of germination percentage}

The germination test was carried out according to International Seed Testing Association (ISTA) rules. For each treatment, 100 seeds were put 
into Petridishes. The Petridishes were put on a laboratory table at room temperature $\left(25 \pm 2^{\circ} \mathrm{C}\right)$. After 8 days, normal, abnormal and diseased seeds were counted. Germination of lentil seed in the laboratory table was $85 \%$. Twenty seeds were sown in each pot. After 24 days germinated seeds were observed and counted. Germination percentage was calculated by the following formula:

Germination $(\%)=$

Number of germinated seeds in each pot

Total number of seeds sown in each pot

\subsection{Crop harvest}

Lentil was harvested after 90 days of sowing. After harvesting root parts were cleaned under running tap water and allow for drying at room temperature. Different growth parameters like root length, shoot length and plant height were measured by using centimeter scale. Plant dry weight, total seed weight, 1000-seed weight, seed yield and stover yield were measured by using digital weight balance and pods plant ${ }^{-1}$ were counted by using hand.

\subsection{Statistical analysis}

Data were statistically analyzed using Analysis of Variance (ANOVA) following CropStat package while the all pair comparisons were done by Statistix 10.

\section{Results and Discussion}

\subsection{Effect of AM inoculation}

The effect of mycorrhizal inoculation on seed germination at 24 DAS, root length $(\mathrm{cm})$, shoot length $(\mathrm{cm})$, root + shoot length $(\mathrm{cm})$, plant dry weight $\left(\mathrm{g}\right.$ plant $\left.{ }^{-1}\right)$, pods plant ${ }^{-1}$, total seed weight $\left(\mathrm{g}\right.$ plant $\left.^{-1}\right)$, 1000-seed weight $(\mathrm{g})$, seed yield $(\mathrm{g}$ $\left.\operatorname{pot}^{-1}\right)$ and stover yield $\left(\mathrm{g} \mathrm{pot}^{-1}\right)$ at harvest have been presented in Table 2 and Table 3 and Figure 2 and Figure 3.

Table 1. Initial fertility status of the soil samples

\begin{tabular}{|c|c|c|c|c|c|c|c|c|c|c|c|c|c|c|}
\hline \multirow{2}{*}{ Soil Properties } & \multirow{2}{*}{ Texture } & \multirow{2}{*}{$\mathrm{pH}$} & \multirow{2}{*}{$\begin{array}{l}\mathrm{OM} \\
(\%)\end{array}$} & $\mathrm{Ca}$ & $\mathrm{Mg}$ & $\mathrm{K}$ & \multirow{2}{*}{$\begin{array}{c}\text { Total N } \\
-(\%)\end{array}$} & $\mathrm{P}$ & S & B & $\mathrm{Cu}$ & $\mathrm{Fe}$ & $\mathrm{Mn}$ & $\mathrm{Zn}$ \\
\hline & & & & me & eq 10 & $0 \mathrm{~g}^{-1}$ & & \multicolumn{7}{|c|}{$\mu \mathrm{g} \mathrm{g}^{-1}$} \\
\hline Result & $\begin{array}{c}\text { Sandy clay } \\
\text { loam }\end{array}$ & 7.6 & 0.32 & 6.6 & 2.3 & 0.09 & 0.017 & 12 & 25 & 0.10 & 1.0 & 14 & 1.3 & 0.85 \\
\hline Critical level & - & - & - & 2.0 & 0.5 & 0.12 & - & 10 & 10 & 0.20 & 0.2 & 4.0 & 1.0 & 0.60 \\
\hline
\end{tabular}

Table 2. Effect of AM on germination (\%), root length, shoot length and root + shoot length of lentil

\begin{tabular}{lcccccccc}
\hline & \multirow{2}{*}{$\begin{array}{c}\text { Germination }(\%) \\
\text { Efter 24 DAS }\end{array}$} & \multicolumn{2}{c}{ Root length } & \multicolumn{2}{c}{ Shoot length } & \multicolumn{2}{c}{$\begin{array}{c}\text { Root + shoot } \\
\text { length }\end{array}$} \\
\cline { 2 - 8 } Effect of AM & \multicolumn{2}{c}{ (cm at harvest) } \\
& $2014-$ & $2015-$ & $2014-$ & $2015-$ & $2014-$ & $2015-$ & $2014-$ & $2014-$ \\
& 2015 & 2016 & 2015 & 2016 & 2015 & 2016 & 2015 & 2015 \\
\hline Without AM & $72.00 \mathrm{~b}$ & $77.25 \mathrm{~b}$ & $4.45 \mathrm{a}$ & $5.39 \mathrm{~b}$ & $16.36 \mathrm{~b}$ & $14.83 \mathrm{~b}$ & $20.81 \mathrm{~b}$ & $20.22 \mathrm{~b}$ \\
With AM & $78.25 \mathrm{a}$ & $85.50 \mathrm{a}$ & $5.12 \mathrm{a}$ & $5.88 \mathrm{a}$ & $17.19 \mathrm{a}$ & $17.10 \mathrm{a}$ & $22.31 \mathrm{a}$ & $22.98 \mathrm{a}$ \\
\hline SE $( \pm)$ & 1.34 & 1.44 & 0.11 & 0.14 & 0.27 & 0.36 & 0.31 & 0.45 \\
F test & $* *$ & $* *$ & $* *$ & $*$ & $*$ & $* *$ & $* *$ & $* *$ \\
\hline
\end{tabular}

AM: Arbuscular mycorrhiza. The values represent means of 04 replicates. Different letters within each column indicate significant differences between treatments. Test CropStat and Statistix 10. **Significant $\mathrm{P} \leq 0.01$, *significant $\mathrm{P} \leq 0.05$ 
Table 3. Effect of AM on plant dry weight, number of pods, total seed weight and 1000-seed weight of lentil

\begin{tabular}{lcccccccc}
\hline & \multicolumn{2}{c}{$\begin{array}{c}\text { Plant dry weight } \\
\text { Effect of AM }\end{array}$} & \multicolumn{2}{c}{$\begin{array}{c}\text { Number of pods } \\
\text { plant }^{-1}\end{array}$} & $\begin{array}{c}\text { Total seed weight } \\
(\mathrm{g} \text { plant }\end{array}$ & & $\begin{array}{c}1000 \text {-seed weight } \\
(\mathrm{g})\end{array}$ \\
& $2014-$ & $2015-$ & $2014-$ & $2015-$ & $2014-$ & $2015-$ & $2014-$ & $2015-$ \\
& 2015 & 2016 & 2015 & 2016 & 2015 & 2016 & 2015 & 2016 \\
\hline Without AM & $0.73 \mathrm{~b}$ & $0.55 \mathrm{~b}$ & $15.82 \mathrm{~b}$ & $11.08 \mathrm{~b}$ & $0.34 \mathrm{~b}$ & $0.26 \mathrm{~b}$ & $15.71 \mathrm{~b}$ & $15.21 \mathrm{~b}$ \\
With AM & $0.95 \mathrm{a}$ & $0.74 \mathrm{a}$ & $17.00 \mathrm{a}$ & $15.09 \mathrm{a}$ & $0.39 \mathrm{a}$ & $0.33 \mathrm{a}$ & $17.73 \mathrm{a}$ & $16.87 \mathrm{a}$ \\
\hline SE $( \pm)$ & 0.23 & 0.15 & 0.40 & 0.29 & 0.82 & 0.76 & 0.39 & 0.28 \\
F test & $* *$ & $* *$ & $*$ & $* *$ & $* *$ & $* *$ & $* *$ & $* *$ \\
\hline
\end{tabular}

AM: Arbuscular mycorrhiza. The values represent means of 04 replicates. Different letters within each column indicate significant differences between treatments. Test CropStat and Statistix 10. **Significant $\mathrm{P} \leq 0.01$, *significant $\mathrm{P} \leq 0.05$

Table 4. Effect of $\mathrm{NaCl}$ on germination (\%), root length, shoot length and root + shoot length of lentil

\begin{tabular}{lcccccccc}
\hline \multirow{2}{*}{$\begin{array}{l}\text { Effect of } \\
\mathrm{NaCl}\end{array}$} & \multicolumn{2}{c}{$\begin{array}{c}\text { Germination }(\%) \\
\text { after } 24 \mathrm{DAS}\end{array}$} & \multicolumn{2}{c}{ Root length } & \multicolumn{2}{c}{ Shoot length } & \multicolumn{2}{c}{ Root + shoot length } \\
\cline { 2 - 8 } & $2014-$ & $2015-$ & $2014-$ & $2015-$ & $2014-$ & $2015-$ & $2014-$ & $2015-$ \\
& 2015 & 2016 & 2015 & 2016 & 2015 & 2016 & 2015 & 2016 \\
\hline $\mathrm{NaCl} \mathrm{0 \%}$ & $94.38 \mathrm{a}$ & $90.63 \mathrm{a}$ & $5.92 \mathrm{a}$ & $6.53 \mathrm{a}$ & $17.76 \mathrm{a}$ & $17.99 \mathrm{a}$ & $23.68 \mathrm{a}$ & $24.52 \mathrm{a}$ \\
$\mathrm{NaCl} \mathrm{1 \%}$ & $84.38 \mathrm{~b}$ & $85.00 \mathrm{ab}$ & $5.27 \mathrm{~b}$ & $6.12 \mathrm{ab}$ & $17.18 \mathrm{ab}$ & $16.66 \mathrm{ab}$ & $22.45 \mathrm{ab}$ & $22.78 \mathrm{ab}$ \\
$\mathrm{NaCl} \mathrm{2 \%}$ & $75.63 \mathrm{c}$ & $81.88 \mathrm{bc}$ & $4.78 \mathrm{bc}$ & $5.66 \mathrm{bc}$ & $16.74 \mathrm{abc}$ & $15.86 \mathrm{bc}$ & $21.52 \mathrm{bc}$ & $21.52 \mathrm{bc}$ \\
$\mathrm{NaCl} \mathrm{3 \%}$ & $65.00 \mathrm{~d}$ & $76.88 \mathrm{~cd}$ & $4.31 \mathrm{c}$ & $5.18 \mathrm{~cd}$ & $16.32 \mathrm{bc}$ & $14.88 \mathrm{c}$ & $20.62 \mathrm{~cd}$ & $20.06 \mathrm{~cd}$ \\
$\mathrm{NaCl} \mathrm{4 \%}$ & $56.25 \mathrm{e}$ & $72.50 \mathrm{~d}$ & $3.65 \mathrm{~d}$ & $4.70 \mathrm{~d}$ & $15.88 \mathrm{c}$ & $14.43 \mathrm{c}$ & $19.53 \mathrm{~d}$ & $19.13 \mathrm{~d}$ \\
\hline $\mathrm{SE}( \pm)$ & 2.12 & 2.28 & 0.17 & 0.22 & 0.42 & 0.56 & 0.49 & 0.71 \\
$\mathrm{~F} \mathrm{test}$ & $* *$ & $* *$ & $* *$ & $* *$ & $*$ & $* *$ & $* *$ & $* *$ \\
\hline
\end{tabular}

The values represent means of 04 replicates. Different letters within each column indicate significant differences between treatments. Test CropStat and Statistix 10. **Significant $\mathrm{P} \leq 0.01$, *significant $\mathrm{P} \leq 0.05$

Table 5. Effect of $\mathrm{NaCl}$ on plant dry weight, no. of pods, total seed weight, and1000-seed weight of lentil

\begin{tabular}{lcccccccc}
\hline \multirow{3}{*}{$\begin{array}{l}\text { Effect of } \\
\mathrm{NaCl}\end{array}$} & \multicolumn{2}{c}{$\begin{array}{c}\text { Plant dry weight } \\
(\mathrm{g} \text { plant }\end{array}$} & \multicolumn{2}{c}{$\begin{array}{c}\text { Number of pods } \\
\text { plant }^{-1}\end{array}$} & $\begin{array}{c}\text { Total seed weight } \\
\left(\mathrm{g} \mathrm{plant}^{-1}\right)\end{array}$ & $\begin{array}{c}1000 \text {-seed weight } \\
(\mathrm{g})\end{array}$ \\
\cline { 2 - 9 } & $2014-$ & $2015-$ & $2014-$ & $2015-$ & $2014-$ & $2015-$ & $2014-$ & $2015-$ \\
$\mathrm{NaCl} \mathrm{0 \%}$ & 2015 & 2016 & 2015 & 2016 & 2015 & 2016 & 2015 & 2016 \\
$\mathrm{NaCl} \mathrm{1 \%}$ & $0.04 \mathrm{a}$ & $0.83 \mathrm{a}$ & $19.20 \mathrm{a}$ & $16.98 \mathrm{a}$ & $0.46 \mathrm{a}$ & $0.40 \mathrm{a}$ & $20.18 \mathrm{a}$ & $17.48 \mathrm{a}$ \\
$\mathrm{NaCl} \mathrm{2 \%}$ & $0.85 \mathrm{bc}$ & $0.66 \mathrm{c}$ & $16.45 \mathrm{bc}$ & $13.15 \mathrm{c}$ & $0.37 \mathrm{c}$ & $0.29 \mathrm{c}$ & $16.65 \mathrm{bc}$ & $16.15 \mathrm{bc}$ \\
$\mathrm{NaCl} \mathrm{3 \%}$ & $0.76 \mathrm{c}$ & $0.55 \mathrm{~d}$ & $15.11 \mathrm{~cd}$ & $11.03 \mathrm{~d}$ & $0.32 \mathrm{~d}$ & $0.25 \mathrm{~d}$ & $15.25 \mathrm{~cd}$ & $15.35 \mathrm{~cd}$ \\
$\mathrm{NaCl} 4 \%$ & $0.65 \mathrm{~d}$ & $0.46 \mathrm{e}$ & $13.55 \mathrm{~d}$ & $8.98 \mathrm{e}$ & $0.27 \mathrm{e}$ & $0.19 \mathrm{e}$ & $13.53 \mathrm{~d}$ & $14.28 \mathrm{~d}$ \\
\hline $\mathrm{SE}( \pm)$ & 0.37 & 0.24 & 0.63 & 0.47 & 0.13 & 0.12 & 0.62 & 0.45 \\
$\mathrm{~F}$ test & $* *$ & $* *$ & $* *$ & $* *$ & $* *$ & $* *$ & $* *$ & $* *$ \\
\hline
\end{tabular}

The values represent means of 04 replicates. Different letters within each column indicate significant differences between treatments. Test CropStat and Statistix 10. **Significant $\mathrm{P} \leq 0.01$ 
Table 6. Interaction effect of $\mathrm{AM}$ and $\mathrm{NaCl}$ on germination (\%), root length, shoot length and root + shoot length of lentil

\begin{tabular}{|c|c|c|c|c|}
\hline \multirow[t]{2}{*}{ Effect of AM } & \multirow{2}{*}{$\begin{array}{l}\text { Germination (\%) } \\
\text { after } 24 \text { DAS }\end{array}$} & Root length & Shoot length & $\begin{array}{c}\text { Root }+ \text { shoot } \\
\text { length }\end{array}$ \\
\hline & & \multicolumn{3}{|c|}{ (cm at harvest) } \\
\hline \multicolumn{5}{|l|}{ 2014-2015 } \\
\hline $\mathrm{NaCl} 0 \%$ & 92.50 & 5.48 & 16.95 & 22.43 \\
\hline $\mathrm{NaCl} 0 \%+\mathrm{AM}$ & 96.25 & 6.35 & 18.58 & 24.93 \\
\hline $\mathrm{NaCl} 1 \%$ & 83.75 & 5.00 & 16.79 & 21.79 \\
\hline $\mathrm{NaCl} 1 \%+\mathrm{AM}$ & 85.00 & 5.55 & 17.75 & 23.12 \\
\hline $\mathrm{NaCl} 2 \%$ & 71.25 & 4.49 & 16.38 & 20.87 \\
\hline $\mathrm{NaCl} 2 \%+\mathrm{AM}$ & 80.00 & 5.07 & 17.10 & 22.17 \\
\hline $\mathrm{NaCl} 3 \%$ & 58.75 & 4.01 & 16.05 & 20.06 \\
\hline $\mathrm{NaCl} 3 \%+\mathrm{AM}$ & 71.25 & 4.60 & 16.59 & 21.19 \\
\hline $\mathrm{NaCl} 4 \%$ & 53.75 & 3.25 & 15.65 & 18.90 \\
\hline $\mathrm{NaCl} 4 \%+\mathrm{AM}$ & 58.75 & 4.05 & 16.11 & 20.16 \\
\hline $\mathrm{SE}( \pm)$ & 3.00 & 0.25 & 0.59 & 0.70 \\
\hline F test & NS & NS & NS & NS \\
\hline $\mathrm{CV}(\%)$ & 8.0 & 10.3 & 7.1 & 6.5 \\
\hline \multicolumn{5}{|l|}{$2015-2016$} \\
\hline $\mathrm{NaCl} \mathrm{0 \%}$ & 88.75 & 6.29 & 16.78 & 23.06 \\
\hline $\mathrm{NaCl} 0 \%+\mathrm{AM}$ & 92.50 & 6.78 & 19.20 & 25.98 \\
\hline $\mathrm{NaCl} 1 \%$ & 81.25 & 5.90 & 15.76 & 21.66 \\
\hline $\mathrm{NaCl} 1 \%+\mathrm{AM}$ & 88.75 & 6.35 & 17.57 & 23.91 \\
\hline $\mathrm{NaCl} 2 \%$ & 77.50 & 5.54 & 14.72 & 20.26 \\
\hline $\mathrm{NaCl} 2 \%+\mathrm{AM}$ & 86.25 & 5.78 & 17.00 & 22.78 \\
\hline $\mathrm{NaCl} 3 \%$ & 71.25 & 4.92 & 13.85 & 18.77 \\
\hline $\mathrm{NaCl} 3 \%+\mathrm{AM}$ & 82.50 & 5.44 & 15.90 & 21.34 \\
\hline $\mathrm{NaCl} 4 \%$ & 67.50 & 4.33 & 13.03 & 17.36 \\
\hline $\mathrm{NaCl} 4 \%+\mathrm{AM}$ & 77.50 & 5.08 & 15.83 & 20.90 \\
\hline $\mathrm{SE}( \pm)$ & 3.23 & 0.31 & 0.80 & 1.00 \\
\hline $\mathrm{F}$ test & NS & NS & NS & NS \\
\hline $\mathrm{CV}(\%)$ & 7.94 & 10.8 & 10.0 & 9.3 \\
\hline
\end{tabular}

AM: Arbuscular mycorrhiza. The values represent means of 04 replicates. Different letters within each column indicate significant differences between treatments. Test CropStat and Statistix 10. NS non significant

Mycorrhizal inoculation significantly increased germination $(78.25 \%)$ at $24 \mathrm{DAS}$, root length $(5.12 \mathrm{~cm})$, shoot length $(17.19 \mathrm{~cm})$, root + shoot length $(22.31 \mathrm{~cm})$, plant dry weight $\left(0.95 \mathrm{~g}\right.$ plant $^{-}$ $\left.{ }^{1}\right)$, pods $\left(17\right.$ plant $\left.^{-1}\right)$, total seed weight $(0.39 \mathrm{~g}$ plant $\left.^{-1}\right), 1000$-seed weight $(17.73 \mathrm{~g})$, seed yield $\left(4.95 \mathrm{~g} \mathrm{pot}^{-1}\right)$ and stover yield $\left(6.87 \mathrm{~g} \mathrm{pot}^{-1}\right)$ at harvest in 2014-2015 and germination (85.50\%) at 24 DAS, root length $(5.88 \mathrm{~cm})$, shoot length $(17.10 \mathrm{~cm})$, root + shoot length $(22.98 \mathrm{~cm})$, plant dry weight $\left(0.74 \mathrm{~g} \mathrm{plant}^{-1}\right)$, pods (15 plant $\left.^{-1}\right)$, total seed weight $\left(0.33 \mathrm{~g} \mathrm{plant}^{-1}\right), 1000$-seed weight $(16.87 \mathrm{~g})$, seed yield $\left(4.30 \mathrm{~g} \mathrm{pot}^{-1}\right)$ and stover yield $\left(5.45 \mathrm{~g} \mathrm{pot}^{-1}\right)$ at harvest in 2015 2016 (Table 2 and Table 3 and Figure 2 and Figure 3).

\subsection{Effect of $\mathrm{NaCl}$}

Effects of different concentration of $\mathrm{NaCl}$ on lentil have been presented in Table 4 and Table 5 and Figure 4 and Figure 5. Significant differences were found in case of germination 
(\%) at 24 DAS, root length $(\mathrm{cm})$, shoot length $(\mathrm{cm})$, root + shoot length $(\mathrm{cm})$, plant dry weight $\left(\mathrm{g}\right.$ plant $\left.^{-1}\right)$, pods plant ${ }^{-1}$, total seed weight $(\mathrm{g}$ plant $\left.{ }^{-1}\right), 1000$-seed weight $(\mathrm{g})$, seed yield $\left(\mathrm{g} \mathrm{pot}^{-}\right.$ $\left.{ }^{1}\right)$ and stover yield $\left(\mathrm{g} \mathrm{pot}^{-1}\right)$.

The highest germination (94.38\%) at 24 DAS, root length $(5.92 \mathrm{~cm})$, shoot length $(17.76 \mathrm{~cm})$, root + shoot length $(23.68 \mathrm{~cm})$, plant dry weight (1.04 g plant $\left.^{-1}\right)$, pods $\left(19.20\right.$ plant $\left.^{-1}\right)$, total seed weight $\left(0.46\right.$ g plant $\left.^{-1}\right), 1000$-seed weight (20.18 $\mathrm{g})$, seed yield $\left(6.62 \mathrm{~g} \mathrm{pot}^{-1}\right)$ and stover yield (8.26 $\left.\mathrm{g} \mathrm{pot}^{-1}\right)$ in 2014-2015 and germination $(90.63 \%)$ at $24 \mathrm{DAS}$, root length $(6.53 \mathrm{~cm})$, shoot length $(17.99 \mathrm{~cm})$, root + shoot length $(24.52 \mathrm{~cm})$, plant dry weight $\left(0.83 \mathrm{~g} \mathrm{plant}^{-1}\right)$, pods $\left(16.98\right.$ plant $\left.^{-1}\right)$, total seed weight $(0.40 \mathrm{~g}$ plant $\left.^{-1}\right), 1000$-seed weight $(17.48 \mathrm{~g})$, seed yield $\left(5.00 \mathrm{~g} \mathrm{pot}^{-1}\right)$ and stover yield $\left(7.03 \mathrm{~g} \mathrm{pot}^{-1}\right)$ in 2015-2016 were observed in $\mathrm{NaCl} 0 \%$ treatment (Table 4 and Table 5 and Figure 4 and Figure 5). The lowest germination $(56.25 \%)$ at 24 DAS, root length $(3.65 \mathrm{~cm})$, shoot length $(15.88 \mathrm{~cm})$, root + shoot length $(19.53 \mathrm{~cm})$, plant dry weight (0.65 $\left.\mathrm{g} \mathrm{plant}^{-1}\right)$, pods $\left(13.55\right.$ plant $\left.^{-1}\right)$, total seed weight $\left(0.27 \mathrm{~g} \mathrm{plant}^{-1}\right), 1000$-seed weight (13.53 $\mathrm{g})$, seed yield $\left(2.76 \mathrm{~g} \mathrm{pot}^{-1}\right)$ and stover yield (3.67 $\mathrm{g} \mathrm{pot}^{-1}$ ) in 2014-2015 and germination $(72.50 \%)$ at $24 \mathrm{DAS}$, root length $(4.70 \mathrm{~cm})$, shoot length $(14.43 \mathrm{~cm})$, root + shoot length $(19.13 \mathrm{~cm})$, plant dry weight $\left(0.46 \mathrm{~g} \mathrm{plant}^{-1}\right)$, pods $\left(8.98\right.$ plant $\left.^{-1}\right)$, total seed weight $(0.19 \mathrm{~g}$ plant $\left.^{-1}\right), 1000$-seed weight (14.28 g), seed yield $\left(2.20 \mathrm{~g} \mathrm{pot}^{-1}\right)$, and stover yield $\left(2.64 \mathrm{~g} \mathrm{pot}^{-1}\right)$ in 2015-2016 were observed in $\mathrm{NaCl} 4 \%$ treatment (Tables 4-5 and Figures 3-4). The highest germination (\%) at $24 \mathrm{DAS}$, root length at harvest, plant dry weight $\left(\mathrm{g}\right.$ plant $\left.{ }^{-1}\right)$, total seed weight $\left(\mathrm{g} \mathrm{plant}^{-1}\right), 1000$-seed weight $(\mathrm{g})$, seed yield $\left(\mathrm{g} \mathrm{pot}^{-1}\right)$ and stover yield $\left(\mathrm{g} \mathrm{pot}^{-1}\right)$ in 20142015 were found in $\mathrm{NaCl} 0 \%$ level which was significantly higher over all other $\mathrm{NaCl}$ levels. In contrast, the highest germination (\%) at $24 \mathrm{DAS}$, root length at harvest $(\mathrm{cm})$, shoot length at harvest $(\mathrm{cm})$, root + shoot length at harvest $(\mathrm{cm})$ and 1000-seed weight $(\mathrm{g})$ in 2015-2016 were found in $\mathrm{NaCl} 0 \%$ level which was significantly higher than $\mathrm{NaCl} 2 \%, \mathrm{NaCl} 3 \%$ and $\mathrm{NaCl} 4 \%$ level but identical to $\mathrm{NaCl} 1 \%$ level. The highest shoot length $(\mathrm{cm})$ in 2014-2015 was found in $\mathrm{NaCl} 0 \%$ level which was significantly higher than $\mathrm{NaCl} 3 \%$ and $\mathrm{NaCl} 4 \%$ level but identical to $\mathrm{NaCl} 1 \%$ and $\mathrm{NaCl} 2 \%$ level and root + shoot length $(\mathrm{cm})$ was found in $\mathrm{NaCl} 0 \%$ level which was significantly higher than $\mathrm{NaCl} 2 \%, \mathrm{NaCl} 3 \%$ and $\mathrm{NaCl} 4 \%$ level but identical to $\mathrm{NaCl} 1 \%$ level. On the other hand, the highest plant dry weight $\left(\mathrm{g} \mathrm{plant}^{-1}\right)$, pod plant ${ }^{-1}$, total seed weight $\left(\mathrm{g}\right.$ plant $\left.{ }^{-1}\right)$, seed yield $\left(\mathrm{g} \mathrm{pot}^{-1}\right)$ and stover yield $(\mathrm{g}$ pot $^{-1}$ ) in 2015-2016 were found in $\mathrm{NaCl} 0 \%$ level which was significantly higher than all other $\mathrm{NaCl}$ levels.

\subsection{Interaction effects of mycorrhizal inoculation and $\mathrm{NaCl}$}

Interaction effects of mycorrhizal inoculation and $\mathrm{NaCl}$ on germination (\%) at $24 \mathrm{DAS}$, root length $(\mathrm{cm})$, shoot length $(\mathrm{cm})$, root + shoot length $(\mathrm{cm})$, plant dry weight $\left(\mathrm{g}_{\text {plant }}{ }^{-1}\right)$, pods plant $^{-1}$, total seed weight $\left(\mathrm{g}\right.$ plant $\left.{ }^{-1}\right), 1000$-seed weight $(\mathrm{g})$, seed yield $\left(\mathrm{g} \mathrm{pot}^{-1}\right)$, and stover yield $\left(\mathrm{g} \mathrm{pot}^{-1}\right)$ were appeared to be statistically nonsignificant (Table 6 and Table 7). This result suggests that mycorrhizal inoculation and $\mathrm{NaCl}$ acted independently. Nevertheless, $\mathrm{NaCl} 0 \%+$ AM combinations resulted in higher germination $\%$, growth and yield of lentil. The highest germination $(96.25 \%$ in $2014-2015$ and $92.50 \%$ in 2015-2016), seed yield (6.45 $\mathrm{g} \mathrm{pot}^{-1}$ in 20142015 and $5.89 \mathrm{~g} \mathrm{pot}^{-1}$ in 2015-2016), and stover yield (9.55 $\mathrm{g} \mathrm{pot}^{-1}$ in 2014-2015 and $8.58 \mathrm{~g} \mathrm{pot}^{-1}$ in 2015-2016) was found in $\mathrm{NaCl} 0 \%+\mathrm{AM}$ treatment. The lowest germination $\%$, seed yield and stover yield was found in $\mathrm{NaCl} 4 \%$ treatment. Mycorrhizal inoculation increased seed yield on an average by $31.85 \%$ during 2014-2015 and $63.71 \%$ during 2015-2016, and increased stover yield on an average by $48.56 \%$ during 2014-2015 and 63.55\% during 2015-2016 over non-mycorrhizal inoculation. A nonsignificant result does not mean that there is no effect of mycorrhizal inoculation and $\mathrm{NaCl}$ but it means that there is not sufficient evidence in my dataset to conclude that there is an effect of mycorrhizal inoculation and $\mathrm{NaCl}$. 
Table 7. Interaction effect of $\mathrm{AM}$ and $\mathrm{NaCl}$ on plant dry weight, no. of pods, total seed weight, 1000seed weight, seed yield and stover yield of lentil

\begin{tabular}{|c|c|c|c|c|c|c|}
\hline Treatments & $\begin{array}{l}\text { Plant dry } \\
\text { weight } \\
\left(\mathrm{g} \text { plant }^{-1}\right)\end{array}$ & $\begin{array}{c}\text { Number of } \\
\text { pods } \\
\text { plant }^{-1}\end{array}$ & $\begin{array}{c}\text { Total seed } \\
\text { weight } \\
\left(\mathrm{g} \text { plant }^{-1}\right)\end{array}$ & $\begin{array}{c}\text { 1000-seed } \\
\text { weight } \\
\text { (g) }\end{array}$ & $\begin{array}{l}\text { Seed yield (g } \\
\left.\operatorname{pot}^{-1}\right)\end{array}$ & $\begin{array}{l}\text { Stover yield } \\
\quad\left(\mathrm{g} \mathrm{pot}^{-1}\right)\end{array}$ \\
\hline \multicolumn{7}{|l|}{ 2014-2015 } \\
\hline $\mathrm{NaCl} \mathrm{0 \%}$ & 0.94 & 18.35 & 0.44 & 18.70 & 6.19 & 6.97 \\
\hline $\mathrm{NaCl} 0 \%+\mathrm{AM}$ & 1.14 & 20.05 & 0.48 & 21.65 & 6.45 & 9.55 \\
\hline $\mathrm{NaCl} 1 \%$ & 0.82 & 17.00 & 0.40 & 16.70 & 4.66 & 5.56 \\
\hline $\mathrm{NaCl} 1 \%+\mathrm{AM}$ & 1.02 & 18.45 & 0.44 & 19.30 & 6.01 & 7.97 \\
\hline $\mathrm{NaCl} 2 \%$ & 0.74 & 15.85 & 0.35 & 15.60 & 3.74 & 4.61 \\
\hline $\mathrm{NaCl} 2 \%+\mathrm{AM}$ & 0.96 & 17.05 & 0.40 & 17.70 & 4.78 & 6.87 \\
\hline $\mathrm{NaCl} 3 \%$ & 0.65 & 14.85 & 0.28 & 14.45 & 2.70 & 3.87 \\
\hline $\mathrm{NaCl} 3 \%+\mathrm{AM}$ & 0.86 & 15.38 & 0.36 & 16.05 & 4.20 & 5.24 \\
\hline $\mathrm{NaCl} 4 \%$ & 0.51 & 13.05 & 0.25 & 13.1 & 2.27 & 2.64 \\
\hline $\mathrm{NaCl} 4 \%+\mathrm{AM}$ & 0.78 & 14.05 & 0.29 & 13.95 & 3.24 & 4.70 \\
\hline$\overline{\mathrm{SE}}( \pm)$ & 0.52 & 0.89 & 0.18 & 0.88 & 0.29 & 0.34 \\
\hline F test & NS & NS & NS & NS & NS & NS \\
\hline $\mathrm{CV}(\%)$ & 12.3 & 10.8 & 9.9 & 10.5 & 13.2 & 11.9 \\
\hline \multicolumn{7}{|l|}{ 2015-2016 } \\
\hline $\mathrm{NaCl} \mathrm{0 \%}$ & 0.70 & 15.15 & 0.36 & 16.60 & 4.12 & 5.49 \\
\hline $\mathrm{NaCl} 0 \%+\mathrm{AM}$ & 0.96 & 18.80 & 0.44 & 18.35 & 5.89 & 8.58 \\
\hline $\mathrm{NaCl} 1 \%$ & 0.64 & 13.50 & 0.30 & 15.95 & 3.72 & 4.56 \\
\hline $\mathrm{NaCl} 1 \%+\mathrm{AM}$ & 0.83 & 17.10 & 0.37 & 17.95 & 5.15 & 6.16 \\
\hline $\mathrm{NaCl} 2 \%$ & 0.59 & 10.80 & 0.27 & 14.95 & 2.82 & 3.36 \\
\hline $\mathrm{NaCl} 2 \%+\mathrm{AM}$ & 0.74 & 15.50 & 0.31 & 17.35 & 3.94 & 4.78 \\
\hline $\mathrm{NaCl} 3 \%$ & 0.47 & 8.55 & 0.21 & 14.65 & 1.98 & 2.13 \\
\hline $\mathrm{NaCl} 3 \%+\mathrm{AM}$ & 0.63 & 13.50 & 0.29 & 16.05 & 3.49 & 4.35 \\
\hline $\mathrm{NaCl} 4 \%$ & 0.35 & 7.40 & 0.16 & 13.90 & 1.37 & 1.89 \\
\hline $\mathrm{NaCl} 4 \%+\mathrm{AM}$ & 0.57 & 10.55 & 0.23 & 14.65 & 3.03 & 3.40 \\
\hline $\mathrm{SE}( \pm)$ & 0.35 & 0.66 & 0.17 & 0.63 & 0.19 & 0.25 \\
\hline$F$ test & NS & NS & NS & NS & NS & NS \\
\hline $\mathrm{CV}(\%)$ & 10.7 & 10.1 & 11.6 & 7.9 & 10.6 & 11.4 \\
\hline
\end{tabular}

AM: Arbuscular mycorrhiza. The values represent means of 04 replicates. Different letters within each column indicate significant differences between treatments. Test CropStat and Statistix 10. NS non significant.

The length of shoot and root decreased with the increasing concentration of $\mathrm{NaCl}$ and the results are in accordance with Rohanipoor et al. (2013) who also reported decrease in shoot length under salt stress in maize. Ahmad et al. (2014) also observed decrease in shoot length with increasing concentration of salt in mulberry. In response to salt stress, reduction in root growth of tomato (Latef and Chaoxing, 2011) and Jatropha curcas (Kumar et al., 2010) has been reported even when the plants were inoculated with the AM fungi. Similar results were also reported by Hajbagheri and Enteshari (2011). In this study, root dry weight increased due to enhance salinity and root fresh weight decreased due to reduced osmotic potential of soil and also due to its low water absorption capacity (Hajbagheri and Enteshari, 2011). Mycorrhizal fungal fibers entering the plants increase cytokinin content resulting in higher water 
absorption and formation of extensive root system in plants. Other group of fibers presented outside the root system produces organic acids solubilizing phosphorus like malic acid, thereby enhancing phosphorus absorption and hence plant dry matter. Phosphorus plays a crucial role in cellular division by regulating the activity of growth hormones. Growth and biomass inhibition under salt stress is reported by Siddiqui et al. (2009) due to high accumulation of $\mathrm{NaCl}$ salt. Our results of decreasing plant dry weight correspond to the findings of Ahmad et al. (2012) on different cultivars of Brassica juncea. Colla et al. (2008) reported improved growth, yield, water status, nutrient content and quality of fruits of Cucurbita pepo plants colonized by Glomus intraradices when exposed to salinity stress. Therefore, enhanced growth of arbuscular mycorrhizal plants has been partly attributed to mycorrhizae mediated nutrient acquisition, especially better $\mathrm{P}$ nutrition within the soil plant system.

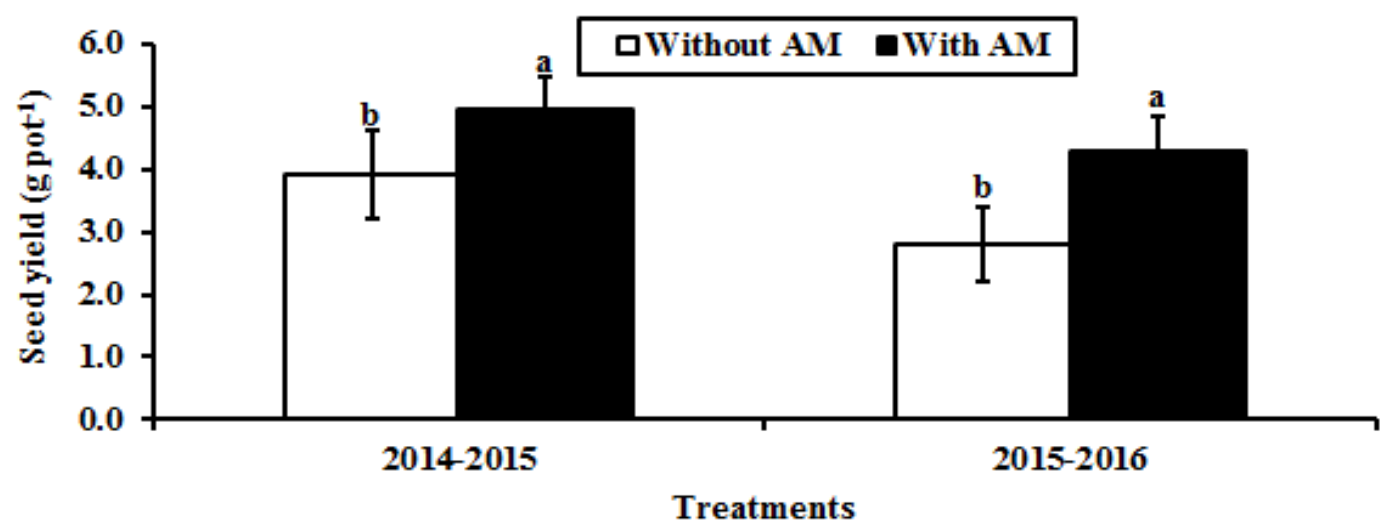

Figure 2. Effect of AM on seed yield $\left(\mathrm{g} \mathrm{pot}^{-1}\right)$ of lentil

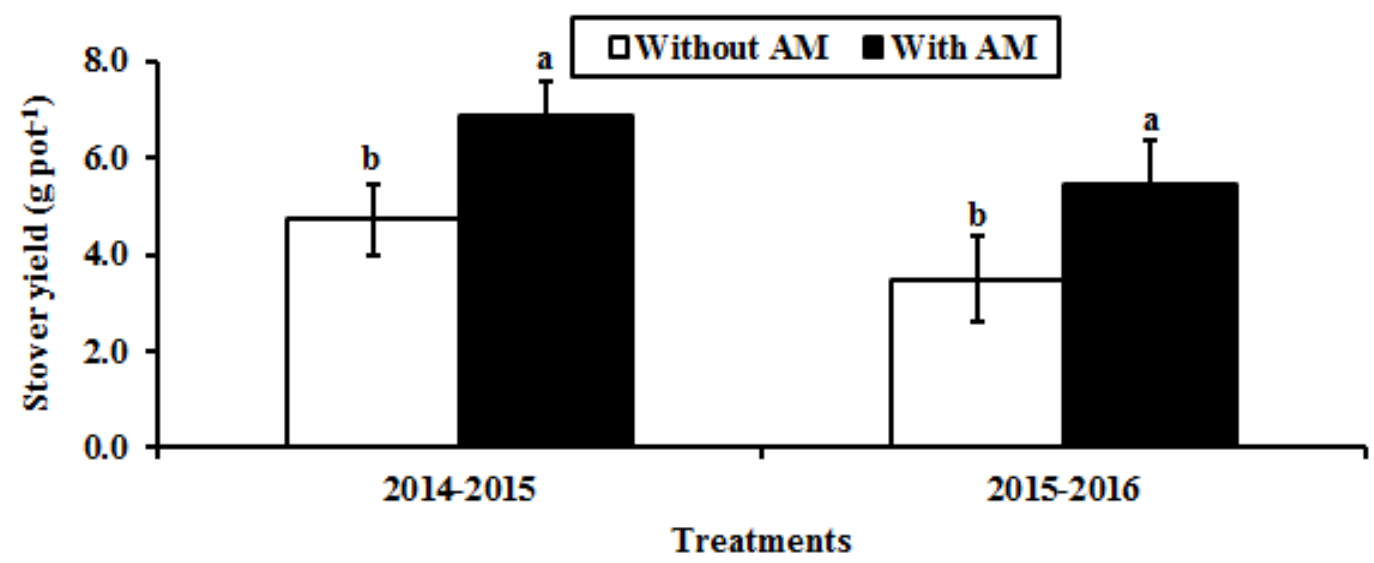

Figure 3. Effect of AM on stover yield $\left(\mathrm{g} \mathrm{pot}^{-1}\right)$ of lentil 


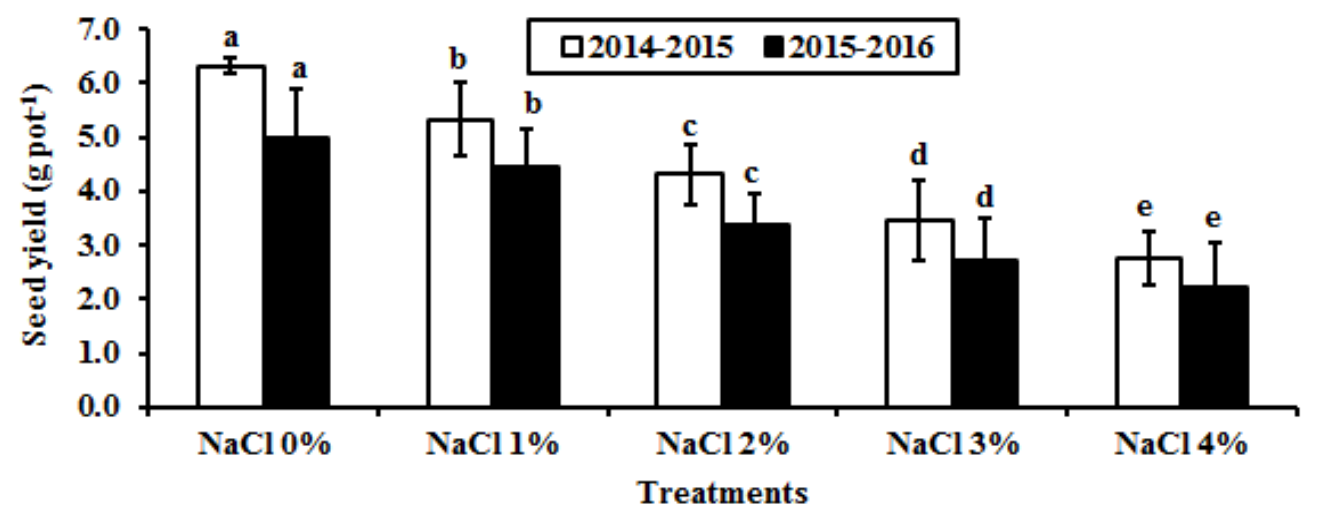

Figure 4. Effect of $\mathrm{NaCl}$ on seed yield $\left(\mathrm{g} \mathrm{pot}^{-1}\right)$ of lentil

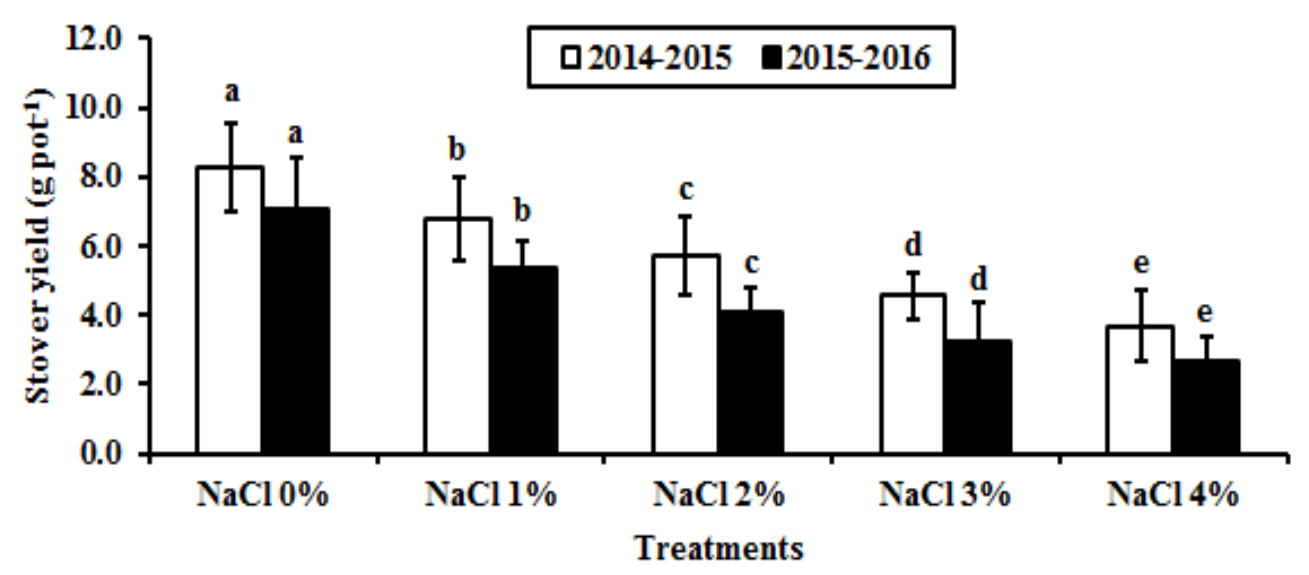

Figure 5. Effect of $\mathrm{NaCl}$ on stover yield $\left(\mathrm{g} \mathrm{pot}^{-1}\right)$ of lentil

Plants inoculated with AM have been reported to improve plant growth and yield even under stress conditions (Wu et al., 2010; Alqarawi et al., 2014). Past research showed the higher growth of mycorrhizal plants under salt stress (Zuccarini and Okurowska, 2008). Hajbagheri and Enteshari (2011) reported maximum plant growth and biomass under salt stress. Ashoori et al. (2015) observed that mycorrhizal Ocimum basilicum plants in saline conditions showed better growth than non-inoculated plants. The results of Hashem et al. (2015) confirmed that $\mathrm{NaCl}$ imposes threat to the survival of tomato plants and application of AM mitigates the negative effect to an appreciable level. Phosphorus limits plant growth due to its poor mobility in the soil. However, its increased availability due to AM fungi symbiosis with the host plant has been reported to enhance plant growth and biomass. Ying-Ning et al. (2013) observed that citrus plants inoculated with AM overcome the adverse effects of $\mathrm{NaCl}$ stress. 

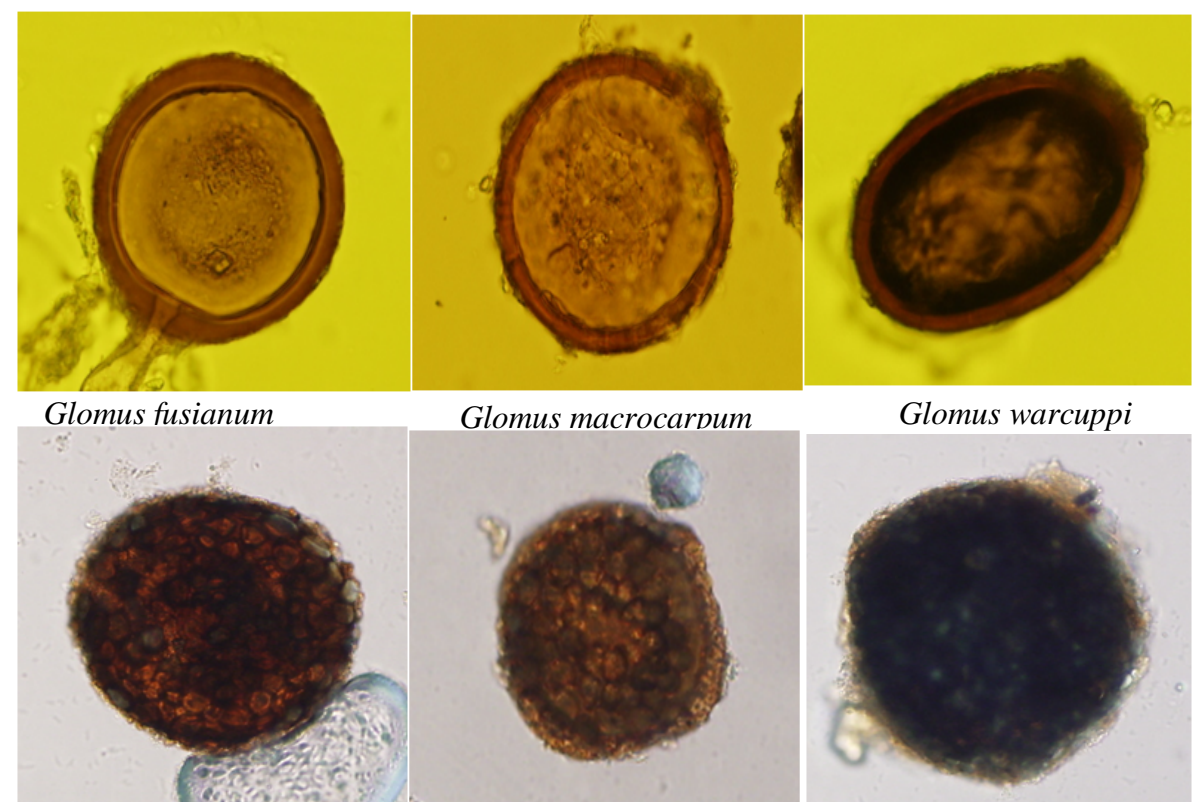

Glomus warcuppi

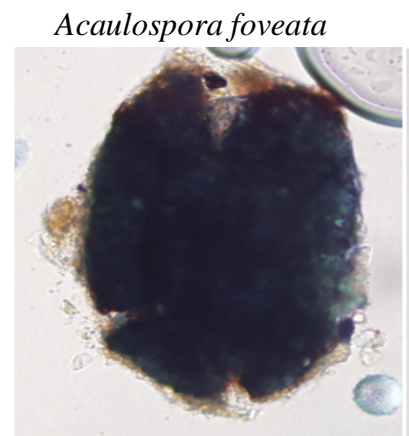

Acaulospora denticulate
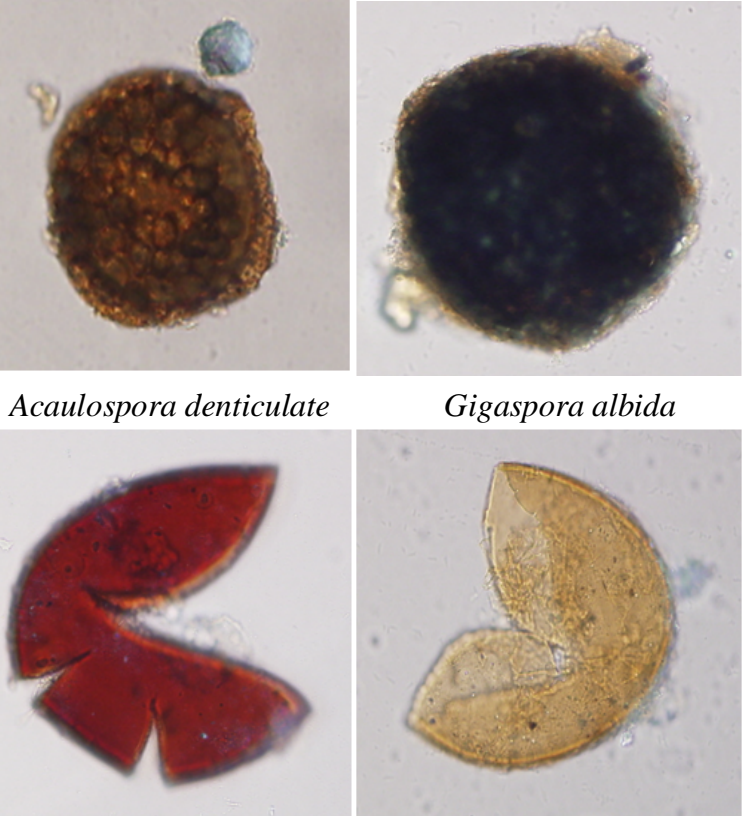

Gigaspora albida

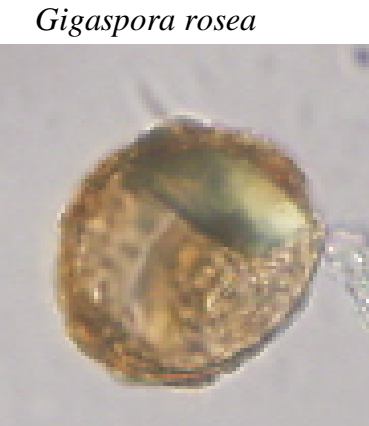

Not identified
Gigaspora spp.

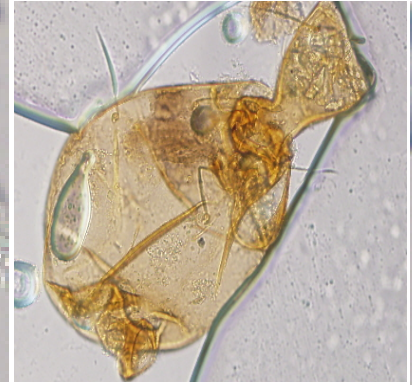

Not identified

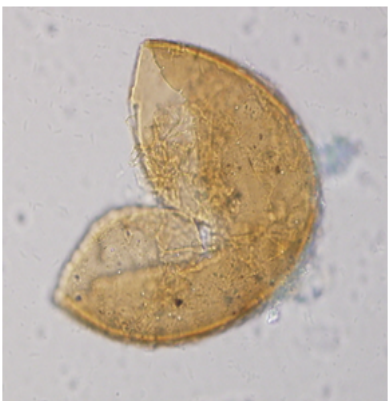

Gigaspora spp.

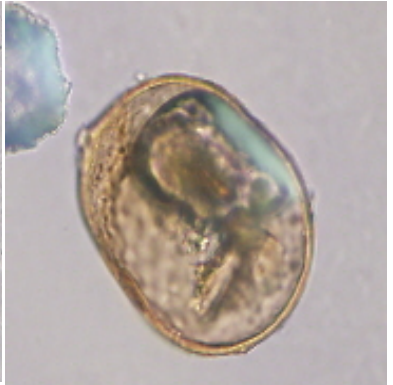

Not identified

Figure 1. Different mycorrhizal spore identified in the Soil Microbiology Laboratory, Soil Science Division, BARI and used for the experiment 
The work of Yamato et al., 2008 and those of Daei et al., 2009 showed that AM fungi can grow naturally in various saline environments where they can improve plant growth. Application of $\mathrm{NaCl}$ significantly reduced growth responses, flower parameters, mineral contents, and levels of mycorrhizal colonization of mycorrhizal and non-mycorrhizal kalanchoe plants comparing to control plants, mainly at high concentrations (Abdul-Wasea et al., 2014). Several researchers have reported that AM inoculated plants grow better than noninoculated plants under salt stress (Zuccarini and Okurowska, 2008). Therefore, the enhanced growth of AM fungi treated plants is due to adequate supply of mineral nutrients, particularly phosphorous, increase in surface area for absorption which is mediated by profound branching of mycorrhizal hyphae and help uptake of water from soil with low water potential.

\section{Conclusions}

Results of the experiment revealed that mycorrhizal plants showed better performance in terms of lentil seed germination (\%) at 24 DAS, growth parameters as well as seed and stover yield $\left(\mathrm{g} \mathrm{pot}^{-1}\right)$. With the increase of $\mathrm{NaCl}$ concentration, germination (\%) at 24 DAS, growth parameters as well as seed and stover yield $\left(\mathrm{g} \mathrm{pot}^{-1}\right)$ decreased significantly. Interaction effects of mycorrhizal inoculation and $\mathrm{NaCl}$ on germination $\%$, growth and yield of lentil were appeared to be statistically non-significant. The highest germination $(96.25 \%$ in $2014-2015$ and $92.50 \%$ in 2015-2016), seed yield (6.45 $\mathrm{g} \mathrm{pot}^{-1}$ in 2014-2015 and 5.89 $\mathrm{g} \mathrm{pot}^{-1}$ in 2015-2016), and stover yield (9.55 $\mathrm{g} \mathrm{pot}^{-1}$ in 2014-2015 and 8.58 $\mathrm{g} \mathrm{pot}^{-1}$ in 2015-2016) was found in $\mathrm{NaCl} 0 \%+$ AM treatment. The lowest germination $\%$, seed yield and stover yield was found in $\mathrm{NaCl} 4 \%$ treatment. Mycorrhizal inoculation increased seed yield on an average by $31.85 \%$ during 2014-2015 and $63.71 \%$ during 2015-2016, and increased stover yield on an average by $48.56 \%$ during 2014-2015 and $63.55 \%$ during 2015-2016 over non-mycorrhizal inoculation. Although,
$\mathrm{NaCl}$ uptake and chemical transformation by seedlings were not tested in this study, these findings gave an indication that the use of effective AM inoculants may help the host plants in increasing overall above ground productivity and plant establishment. Further, research efforts should be oriented to know the direct or indirect relationship of $\mathrm{AM}$ and $\mathrm{NaCl}$ with belowground and above-ground community via plants. Conclusively, efforts should be made in such a way for improving the quality of $\mathrm{AM}$ as a complete commercial supplement for lentil in saline areas which have the potential to reclaim a saline soil owing to enhancement of productivity for a sustainable agriculture as well as a green, safe and food secure world.

\section{References}

Abdul-Wasea, A. A., Abdel-Fattah, G. M., Elhindi, K. M. and Abdel-Salam E. M. 2014. The impact of arbuscular mychorrhizal fungi in improving growth, flower yield and tolerance of kalanchoe (Kalanchoe blossfeldiana Poelin) plants grown in NaCl-stress conditions. Journal of Food Agriculture and Environment, 12(1): 105-112.

Ahmad, P., Hakeem, K. R., Kumar, A., Ashraf, M. and Akram, N. A. 2012. Salt-induced changes in photosynthetic activity and oxidative defense system of three cultivars of mustard (Brassica juncea L.). African Journal of Biotechnology, 11(11): 2694-2703.

Ahmad, P., Ozturk, M., Sharma, S. and Gucel, S. 2014. Effect of sodium carbonate-induced salinity-alkalinity on some key osmoprotectants, protein profile, antioxidant enzymes, and lipid peroxidation in two mulberry (Morus alba L.) cultivars. Journal of Plant Interactions, 9(1): 460-467.

Alqarawi, A. A., Abd_Allah, E. F. and Hashem, A. 2014. Alleviation of salt-induced adverse impact via mycorrhizal fungi in 
Ephedra aphylla Forssk. Journal of Plant Interaction, 9(1): 802-810.

Ashoori, M., Ashraf, S. and Alipour, Z. T. 2015. Investigating the effect of two species of mycorrhiza fungi and salinity on growth, function and chlorophyll content on Ocimum basilicum. International Journal of Agriculture and Crop Sciences, 8(3): 503-509.

BARC (Bangladesh Agricultural Research Council). 2012. Fertilizer Recommendation Guide. Bangladesh Agricultural Research Council, Farmgate, New Airport Road, Dhaka-1215. 101 pp.

BBS. 2015. Yearbook of Agricultural Statistics $\left(27^{\text {th }}\right.$ Series). Bangladesh Bureau of Statistics. Statistics and Information Division. Ministry of Planning, Government of the People's Republic of Bangladesh, $103 \mathrm{p}$.

Colla, G., Rouphael, Y., Cardarelli, M., Tullio, M., Rivera, C. M. and Rea, E. 2008. Alleviation of salt stress by Arbuscular mycorrhizal in zucchini plants grown at low and high phosphorus concentration. Biology and Fertility of Soils, 44: 501509.

Daei, G., Ardekani, M. R., Rejali, F., Teimuri, S. and Miransari, M. 2009. Alleviation of salinity stress on wheat yield, yield components, and nutrient uptake using arbuscular mycorrhizal fungi under field conditions. Journal of Plant Physiology, 166: 617-625.

Evelin, H., Giri, B. and Kapoor, R. 2012. Contribution of Glomus intraradices inoculation to nutrient acquisition and mitigation of ionic imbalance in $\mathrm{NaCl}$ stressed Trigonella foenum-graecum. Mycorrhiza, 22: 203-217.

Gerdemann, J. W. and Nicolson, T. H. 1963. Species of mycorrhizal endogone species extracted from soil by wet sieving and decanting method. Transactions of the British Mycological Society, 46: 235-246.

Hajbagheri, S. and Enteshari, S. 2011. Effects of mycorrhizal fungi on photosynthetic pigments, root mycorrhizal colonization and morphological characteristics of salt stressed Ocimum basilicum L. Iranian Journal of Plant Physiology, 1(4): 215222.

Hameed, A., Egamberdieva, D., Abd_Allah, E. F., Hashem, A., Kumar, A. and Ahmad, P. 2014. Salinity stress and arbuscular mycorrhizal symbiosis in plants. In: (Ed.): Miransari, M., Use of Microbes for the Alleviation of Soil Stresses (Springer New York). 1: 139-159.

Hashem, A., Abd_Allah, E. F., Alqarawi, A. A., Alwhibi, M. S., Alenazi, M. M., Dilfuza, E. and Ahmad, P. 2015. Arbuscular mycorrhizal fungi mitigates $\mathrm{NaCl}$ induced adverse effects on Solanum lycopersicum L. Pakistan Journal of Botany, 47(1): 327-340.

Jamil, A., Riaz, S., Ashraf, M. and Foolad, M. R. 2011. Gene expression profiling of plants under salt stress. Crit. Rev. Plant Science, 30(5): 435-458.

Kumar, A., Sharma, S. and Mishra, S. 2010. Influence of arbuscular mycorrhizal (AM) fungi and salinity on seedling growth, solute accumulation and mycorrhizal dependency of Jatropha curcas L. Journal of Plant Growth Regulation, 29: 297-306.

Latef, A. H. A. and Chaoxing, H. 2011. Effect of arbuscular mycorrhizal fungi on growth, mineral nutrition, antioxidant enzymes activity and fruit yield of tomato grown under salinity stress. Scientia Horticulturae, 127: 228-233.

Mali, B. S., Thengal, S. S. and Pate, P. N. 2012. Physico-chemical characteristics of salt affected soil from Barhanpur. Indian 
Journal of Biological Research, 3: 40914093.

Rohanipoor, A., Norouzi, M., Moezzi, A. and Hassibi, P. 2013. Effect of silicon on some physiological properties of maize (Zea mays) under salt stress. Journal of Biodiversity and Environmental Sciences, 7(20): 71-79.

Siddiqui, M. H., Mohammad, F. and Khan, M. N. 2009. Morphological and physiobiochemical characterization of Brassica juncea L. Czern. and Coss genotypes under salt stress. Journal of Plant Interactions, 4: 67-80.

SRDI 2010. Saline soils of Bangladesh. SRMAF Project, Ministry of Agriculture, Dhaka, Bangladesh. 1-60 pp.

Wu, Q. S., Zou, Y. N. and Abd_Allah, E. F. 2014. Mycorrhizal Association and ROS in Plants. In: (Ed.): Ahmad, P., Oxidative Damage to Plants. Elsevier Inc. 453-475 pp.
Wu, Q. S., Zou, Y. N., Liu, W., Ye, X. F., Zai, H. F. and Zhao, L. J. 2010. Alleviation of salt stress in citrus seedlings inoculated with mycorrhiza: changes in leaf antioxidant defense systems. Plant, Soil and Environment, 56(10): 470-475.

Yamato, M., Ikeda, S. and Iwase, K. 2008. Community of arbuscular mycorrhizal fungi in coastal vegetation on Okinawa Island and effect of the isolated fungi on growth of sorghum under salt-treated conditions. Mycorrhiza, 18: 241-249.

Ying-Ning, Z., Yong-Chao, L. and Wu, Q. S. 2013. Mycorrhizal and non-mycorrhizal responses to salt stress in trifoliate orange: plant growth, root architecture and soluble sugar accumulation. International Journal of Agriculture and Biology, 15: 565-569.

Zuccarini, P. and Okurowska, P. 2008. Effects of mycorrhizal colonization and fertilization on growth and photosynthesis of sweet basil under salt stress. Journal of Plant Nutrition, 31: 497-513. 